\title{
ANTI-SYNCHRONIZATION OF HYPERCHAOTIC WANG AND HYPERCHAOTIC LI SYSTEMS WITH UNKNOWN PARAMETERS VIA ADAPTIVE CONTROL
}

\author{
Sundarapandian Vaidyanathan ${ }^{1}$ \\ ${ }^{1}$ Research and Development Centre, Vel Tech Dr. RR \& Dr. SR Technical University \\ Avadi, Chennai-600 062, Tamil Nadu, INDIA \\ sundarvtu@gmail. com
}

\begin{abstract}
In chaos theory, the problem anti-synchronization of chaotic systems deals with a pair of chaotic systems called drive and response systems. In this problem, the design goal is to drive the sum of their respective states to zero asymptotically. This problem gets even more complicated and requires special attention when the parameters of the drive and response systems are unknown. This paper uses adaptive control theory and Lyapunov stability theory to derive new results for the anti-synchronization of hyperchaotic Wang system (2008) and hyperchaotic Li system (2005) with uncertain parameters. Hyperchaotic systems are nonlinear dynamical systems exhibiting chaotic behaviour with two or more positive Lyapunov exponents. The hyperchaotic systems have applications in areas like oscillators, lasers, neural networks, encryption, secure transmission and secure communication. The main results derived in this paper are validated and demonstrated with MATLAB simulations.
\end{abstract}

\section{KEYWORDS}

Hyperchaos, Hyperchaotic Systems, Adaptive Control, Anti-Synchronization.

\section{INTRODUCTION}

Hyperchaotic systems are typically defined as nonlinear chaotic systems having two or more positive Lyapunov exponents. They are applicable in several areas like lasers [1], chemical reactions [2], neural networks [3], oscillators [4], data encryption [5], secure communication [68], etc.

In chaos theory, the anti-synchronization problem deals with a pair of chaotic systems called the drive and response systems, where the design goal is to render the respective states to be same in magnitude, but opposite in sign, or in other words, to drive the sum of the respective states to zero asymptotically [9].

There are several methods available in the literature to tackle the problem of synchronization and anti-synchronization of chaotic systems like active control method [10-12], adaptive control method [13-15], backstepping method [16-19], sliding control method [20-22] etc.

This paper derives new results for the adaptive controller design for the anti-synchronization of hyperchaotic Wang systems ([23], 2008) and hyperchaotic Li systems ([24], 2005) with unknown parameters. Lyapunov stability theory [25] has been applied to prove the main results of this paper. Numerical simulations have been shown using MATLAB to illustrate the results.

DOI : 10.5121/ijcseit.2013.3203 
International Journal of Computer Science, Engineering and Information Technology (IJCSEIT), Vol.3, No.2,April2013

\section{The Problem of Anti-Synchronization of Chaotic Systems}

In chaos synchronization problem, the drive system is described by the chaotic dynamics

$$
\dot{x}=A x+f(x)
$$

where $A$ is the $n \times n$ matrix of the system parameters and $f: R^{n} \rightarrow R^{n}$ is the nonlinear part. Also, the response system is described by the chaotic dynamics

$$
\dot{y}=B y+g(y)+u
$$

where $B$ is the $n \times n$ matrix of the system parameters, $g: R^{n} \rightarrow R^{n}$ is the nonlinear part and $u \in R^{n}$ is the active controller to be designed.

For the pair of chaotic systems (1) and (2), the design goal of the anti-synchronization problem is to construct a feedback controller $u$, which anti-synchronizes their states for all $x(0), y(0) \in R^{n}$. The anti-synchronization error is defined as

$$
e=y+x
$$

The error dynamics is obtained as

$$
\dot{e}=B y+A x+g(y)+f(x)+u
$$

The design goal is to find a feedback controller $u$ so that

$$
\lim _{t \rightarrow \infty}\|e(t)\|=0 \text { for all } e(0) \in R^{n}
$$

Using the matrix method, we consider a candidate Lyapunov function

$$
V(e)=e^{T} P e,
$$

where $P$ is a positive definite matrix.

It is noted that $V: R^{n} \rightarrow R$ is a positive definite function.

If we find a feedback controller $u$ so that

$$
\dot{V}(e)=-e^{T} Q e,
$$

where $Q$ is a positive definite matrix, then $\dot{V}: R^{n} \rightarrow R$ is a negative definite function.

Thus, by Lyapunov stability theory [26], the error dynamics (4) is globally exponentially stable. When the system parameters in (1) and (2) are unknown, we apply adaptive control theory to construct a parameter update law for determining the estimates of the unknown parameters. 
International Journal of Computer Science, Engineering and Information Technology (IJCSEIT), Vol.3, No.2,April2013

\section{HYPERCHAOTIC WANG AND HYPERCHAOTIC LI SYSTEMS}

The hyperchaotic Wang system ([23], 2008) is given by

$$
\begin{aligned}
& \dot{x}_{1}=a\left(x_{2}-x_{1}\right)+x_{2} x_{3} \\
& \dot{x}_{2}=c x_{1}-x_{1} x_{3}-x_{2}-0.5 x_{4} \\
& \dot{x}_{3}=-d x_{3}+x_{1} x_{2} \\
& \dot{x}_{4}=b x_{4}+0.5 x_{1} x_{3}
\end{aligned}
$$

where $a, b, c, d$ are constant, positive parameters of the system.

The Wang system (8) depicts a hyperchaotic attractor for the parametric values

$$
a=40, \quad b=1.7, c=88, d=3
$$

The Lyapunov exponents of the system (8) are determined as

$$
\lambda_{1}=3.2553, \quad \lambda_{2}=1.4252, \quad \lambda_{3}=0, \quad \lambda_{4}=-46.9794
$$

Since there are two positive Lyapunov exponents in (10), the Wang system (8) is hyperchaotic for the parametric values (9).

Figure 1 shows the phase portrait of the hyperchaotic Wang system.

The hyperchaotic Li system ([24], 2005) is given by

$$
\begin{aligned}
& \dot{x}_{1}=\alpha\left(x_{2}-x_{1}\right)+x_{4} \\
& \dot{x}_{2}=\delta x_{1}-x_{1} x_{3}+\gamma x_{2} \\
& \dot{x}_{3}=-\beta x_{3}+x_{1} x_{2} \\
& \dot{x}_{4}=x_{2} x_{3}+r x_{4}
\end{aligned}
$$

where $\alpha, \beta, \gamma, \delta, r$ are constant, positive parameters of the system.

The Li system (11) depicts a hyperchaotic attractor for the parametric values

$$
\alpha=35, \quad \beta=3, \quad \gamma=12, \quad \delta=7, \quad r=0.58
$$

The Lyapunov exponents of the system (11) for the parametric values in (12) are

$$
\lambda_{1}=0.5011, \quad \lambda_{2}=0.1858, \quad \lambda_{3}=0, \quad \lambda_{4}=-26.1010
$$

Since there are two positive Lyapunov exponents in (13), the Li system (11) is hyperchaotic for the parametric values (12). Figure 2 shows the phase portrait of the hyperchaotic Li system. 

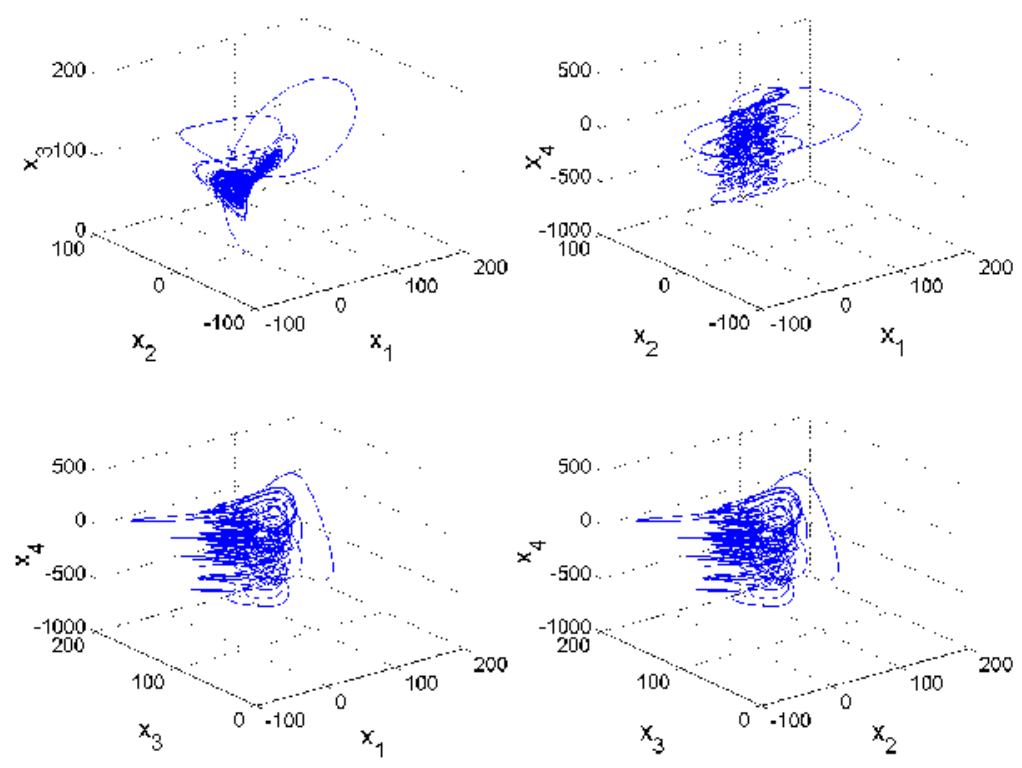

Figure 1. Hyperchaotic Attractor of the Hyperchaotic Wang System
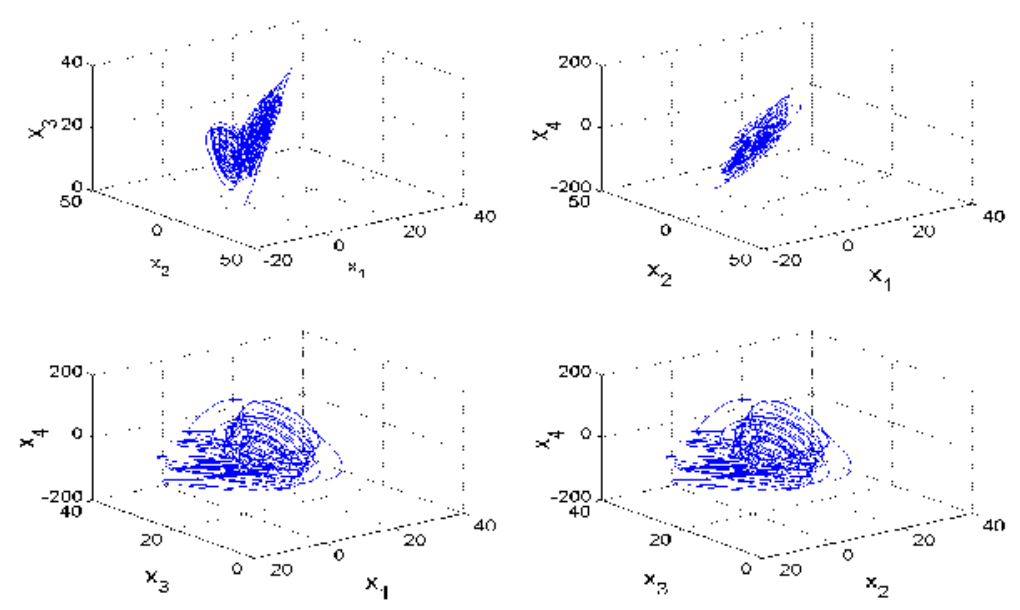

Figure 2. Hyperchaotic Attractor of the Hyperchaotic Li System

\section{Anti-Synchronization of Hyperchaotic Wang Systems Via ADAPTIVE CONTROL}

In this section, we derive new results for designing a controller for the anti-synchronization of identical hyperchaotic Wang systems (2008) with unknown parameters via adaptive control. The drive system is the hyperchaotic Wang dynamics given by 
International Journal of Computer Science, Engineering and Information Technology (IJCSEIT), Vol.3, No.2,April2013

$$
\begin{aligned}
& \dot{x}_{1}=a\left(x_{2}-x_{1}\right)+x_{2} x_{3} \\
& \dot{x}_{2}=c x_{1}-x_{1} x_{3}-x_{2}-0.5 x_{4} \\
& \dot{x}_{3}=-d x_{3}+x_{1} x_{2} \\
& \dot{x}_{4}=b x_{4}+0.5 x_{1} x_{3}
\end{aligned}
$$

where $a, b, c, d$ are unknown parameters of the system and $x \in R^{4}$ is the state. The response system is the controlled hyperchaotic Wang dynamics given by

$$
\begin{aligned}
& \dot{y}_{1}=a\left(y_{2}-y_{1}\right)+y_{2} y_{3}+u_{1} \\
& \dot{y}_{2}=c y_{1}-y_{1} y_{3}-y_{2}-0.5 y_{4}+u_{2} \\
& \dot{y}_{3}=-d y_{3}+y_{1} y_{2}+u_{3} \\
& \dot{y}_{4}=b y_{4}+0.5 y_{1} y_{3}+u_{4}
\end{aligned}
$$

where $y \in R^{4}$ is the state and $u_{1}, u_{2}, u_{3}, u_{4}$ are the adaptive controllers to be designed.

For the anti-synchronization, the error $e$ is defined as

$$
e_{1}=y_{1}+x_{1}, \quad e_{2}=y_{2}+x_{2}, \quad e_{3}=y_{3}+x_{3}, \quad e_{4}=y_{4}+x_{4}
$$

Then we derive the error dynamics as

$$
\begin{aligned}
& \dot{e}_{1}=a\left(e_{2}-e_{1}\right)+y_{2} y_{3}+x_{2} x_{3}+u_{1} \\
& \dot{e}_{2}=c e_{1}-e_{2}-0.5 e_{4}-y_{1} y_{3}-x_{1} x_{3}+u_{2} \\
& \dot{e}_{3}=-d e_{3}+y_{1} y_{2}+x_{1} x_{2}+u_{3} \\
& \dot{e}_{4}=b e_{4}+0.5\left(y_{1} y_{3}+x_{1} x_{3}\right)+u_{4}
\end{aligned}
$$

The adaptive controller to solve the anti-synchronization problem is taken as

$$
\begin{aligned}
& u_{1}=-\hat{a}(t)\left(e_{2}-e_{1}\right)-y_{2} y_{3}-x_{2} x_{3}-k_{1} e_{1} \\
& u_{2}=-\hat{c}(t) e_{1}+e_{2}+0.5 e_{4}+y_{1} y_{3}+x_{1} x_{3}-k_{2} e_{2} \\
& u_{3}=\hat{d}(t) e_{3}-y_{1} y_{2}-x_{1} x_{2}-k_{3} e_{3} \\
& u_{4}=-\hat{b}(t) e_{4}-0.5\left(y_{1} y_{3}+x_{1} x_{3}\right)-k_{4} e_{4}
\end{aligned}
$$

In Eq. (18), $k_{i},(i=1,2,3,4)$ are positive gains and $\hat{a}(t), \hat{b}(t), \hat{c}(t), \hat{d}(t)$ are estimates for the unknown parameters $a, b, c, d$ respectively.

By the substitution of (18) into (17), the error dynamics is obtained as

$$
\begin{aligned}
& \dot{e}_{1}=(a-\hat{a}(t))\left(e_{2}-e_{1}\right)-k_{1} e_{1} \\
& \dot{e}_{2}=(c-\hat{c}(t)) e_{1}-k_{2} e_{2} \\
& \dot{e}_{3}=-(d-\hat{d}(t)) e_{3}-k_{3} e_{3} \\
& \dot{e}_{4}=(b-\hat{b}(t)) e_{4}-k_{4} e_{4}
\end{aligned}
$$


International Journal of Computer Science, Engineering and Information Technology (IJCSEIT), Vol.3, No.2,April2013

Next, we define the parameter estimation errors as

$$
e_{a}(t)=a-\hat{a}(t), e_{b}(t)=b-\hat{b}(t), e_{c}(t)=c-\hat{c}(t), e_{d}(t)=d-\hat{d}(t)
$$

Upon differentiation, we get

$$
\dot{e}_{a}(t)=-\dot{\hat{a}}(t), \dot{e}_{b}(t)=-\dot{\hat{b}}(t), \dot{e}_{c}(t)=-\dot{\hat{c}}(t), \dot{e}_{d}(t)=-\dot{\hat{d}}(t)
$$

Substituting (20) into the error dynamics (19), we obtain

$$
\begin{aligned}
& \dot{e}_{1}=e_{a}\left(e_{2}-e_{1}\right)-k_{1} e_{1} \\
& \dot{e}_{2}=e_{c} e_{1}-k_{2} e_{2} \\
& \dot{e}_{3}=-e_{d} e_{3}-k_{3} e_{3} \\
& \dot{e}_{4}=e_{b} e_{4}-k_{4} e_{4}
\end{aligned}
$$

We consider the candidate Lyapunov function

$$
V=\frac{1}{2}\left(e_{1}^{2}+e_{2}^{2}+e_{3}^{2}+e_{4}^{2}+e_{a}^{2}+e_{b}^{2}+e_{c}^{2}+e_{d}^{2}\right)
$$

Differentiating (23) along the dynamics (21) and (22), we obtain

$$
\begin{aligned}
\dot{V}= & -k_{1} e_{1}^{2}-k_{2} e_{2}^{2}-k_{3} e_{3}^{2}-k_{4} e_{4}^{2}+e_{a}\left[e_{1}\left(e_{2}-e_{1}\right)-\dot{\hat{a}}\right]+e_{b}\left(e_{4}^{2}-\dot{\hat{b}}\right) \\
& +e_{c}\left(e_{1} e_{2}-\dot{\hat{c}}\right)+e_{d}\left(-e_{3}^{2}-\dot{\hat{d}}\right)
\end{aligned}
$$

In view of (24), we choose the following parameter update law:

$$
\begin{aligned}
& \dot{\hat{a}}=e_{1}\left(e_{2}-e_{1}\right)+k_{5} e_{a} \\
& \dot{\hat{b}}=e_{4}^{2}+k_{6} e_{b} \\
& \dot{\hat{c}}=e_{1} e_{2}+k_{7} e_{c} \\
& \dot{\hat{d}}=-e_{3}^{2}+k_{8} e_{d}
\end{aligned}
$$

Next, we prove the following main result of this section.

Theorem 4.1 The adaptive control law defined by Eq. (18) along with the parameter update law defined by Eq. (25), where $k_{i},(i=1,2, \ldots, 8)$ are positive constants, render global and exponential anti-synchronization of the identical hyperchaotic Wang systems (14) and (15) with unknown parameters for all initial conditions $x(0), y(0) \in R^{4}$. In addition, the parameter estimation errors $e_{a}(t), e_{b}(t), e_{c}(t), e_{d}(t)$ globally and exponentially converge to zero for all initial conditions. 
International Journal of Computer Science, Engineering and Information Technology (IJCSEIT), Vol.3, No.2,April2013

Proof. The proof is via Lyapunov stability theory [25] by taking $V$ defined by Eq. (23) as the candidate Lyapunov function. Substituting the parameter update law (25) into (24), we get

$$
\dot{V}(e)=-k_{1} e_{1}^{2}-k_{2} e_{2}^{2}-k_{3} e_{3}^{2}-k_{4} e_{4}^{2}-k_{5} e_{a}^{2}-k_{6} e_{b}^{2}-k_{7} e_{c}^{2}-k_{8} e_{d}^{2}
$$

which is a negative definite function on $R^{8}$. This completes the proof.

Next, we demonstrate our adaptive anti-synchronization results with MATLAB simulations. The classical fourth order R-K method with time-step $h=10^{-8}$ has been used to solve the hyperchaotic Wang systems (14) and (15) with the adaptive controller defined by (18) and parameter update law defined by (25).

The feedback gains in the adaptive controller (18) are taken as $k_{i}=5,(i=1, \ldots, 8)$.

The parameters of the hyperchaotic Wang systems are taken as in the hyperchaotic case, i.e.

$$
a=40, \quad b=1.7, c=88, d=3
$$

For simulations, the initial conditions of the drive system (14) are taken as

$$
x_{1}(0)=37, \quad x_{2}(0)=-16, \quad x_{3}(0)=14, \quad x_{4}(0)=11
$$

Also, the initial conditions of the response system (15) are taken as

$$
y_{1}(0)=21, \quad y_{2}(0)=32, \quad y_{3}(0)=-28, \quad y_{4}(0)=-8
$$

Also, the initial conditions of the parameter estimates are taken as

$$
\hat{a}(0)=12, \hat{b}(0)=4, \hat{c}(0)=-6, \hat{d}(0)=5
$$

Figure 3 depicts the anti-synchronization of the identical hyperchaotic Wang systems.

Figure 4 depicts the time-history of the anti-synchronization errors $e_{1}, e_{2}, e_{3}, e_{4}$.

Figure 5 depicts the time-history of the parameter estimation errors $e_{a}, e_{b}, e_{c}, e_{d}$. 


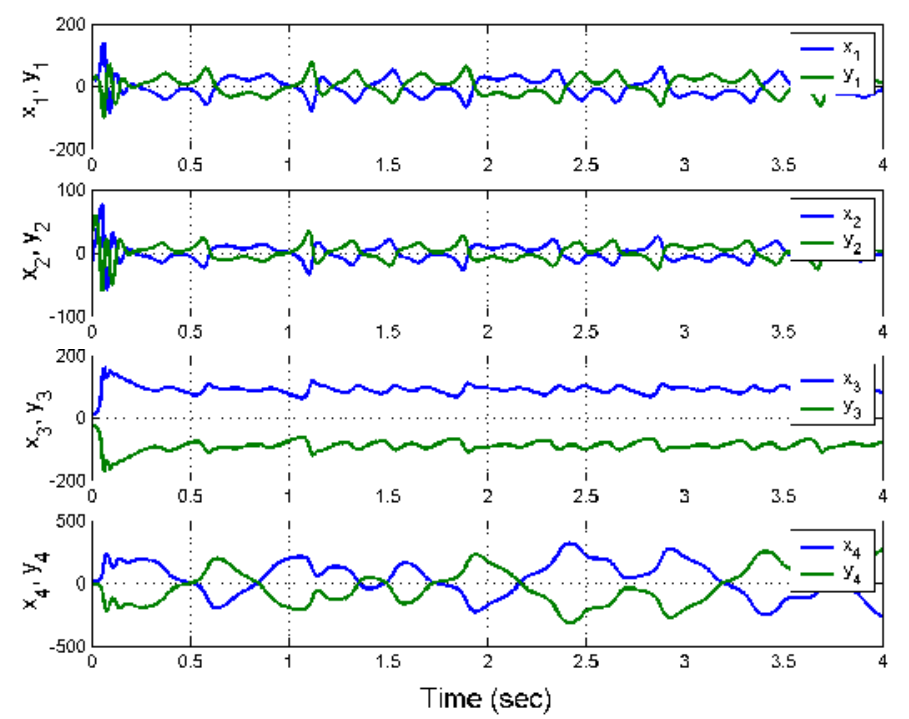

Figure 3. Anti-Synchronization of Identical Hyperchaotic Wang Systems

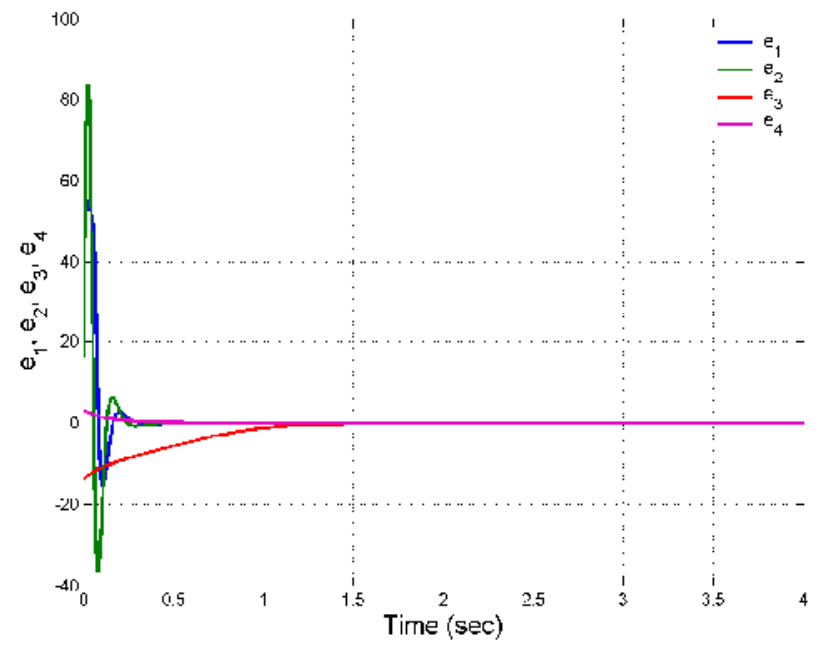

Figure 4. Time-History of the Anti-Synchronization Errors $e_{1}, e_{2}, e_{3}, e_{4}$ 


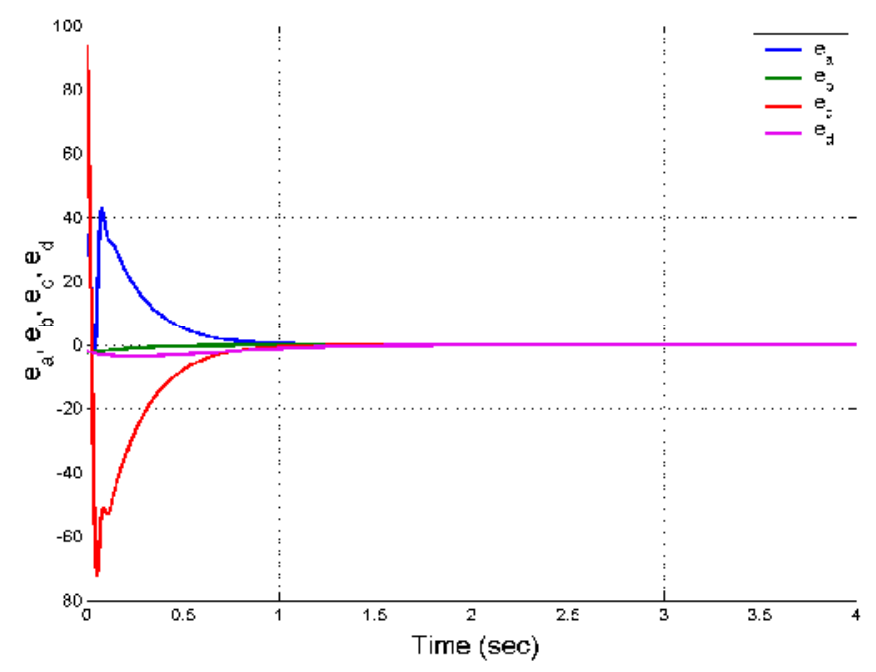

Figure 5. Time-History of the Parameter Estimation Errors $e_{a}, e_{b}, e_{c}, e_{d}$

\section{ANTi-SynChronization OF HyPerchaotic Li Systems Via AdAPTIVE CONTROL}

In this section, we derive new results for designing a controller for the anti-synchronization of identical hyperchaotic Li systems (2005) with unknown parameters via adaptive control. The drive system is the hyperchaotic Li dynamics given by

$$
\begin{aligned}
& \dot{x}_{1}=\alpha\left(x_{2}-x_{1}\right)+x_{4} \\
& \dot{x}_{2}=\delta x_{1}-x_{1} x_{3}+\gamma x_{2} \\
& \dot{x}_{3}=-\beta x_{3}+x_{1} x_{2} \\
& \dot{x}_{4}=x_{2} x_{3}+r x_{4}
\end{aligned}
$$

where $\alpha, \beta, \gamma, \delta, r$ are unknown parameters of the system and $x \in R^{4}$ is the state.

The response system is the controlled hyperchaotic Li dynamics given by

$$
\begin{aligned}
& \dot{y}_{1}=\alpha\left(y_{2}-y_{1}\right)+y_{4}+u_{1} \\
& \dot{y}_{2}=\delta y_{1}-y_{1} y_{3}+\gamma y_{2}+u_{2} \\
& \dot{y}_{3}=-\beta y_{3}+y_{1} y_{2}+u_{3} \\
& \dot{y}_{4}=y_{2} y_{3}+r y_{4}+u_{4}
\end{aligned}
$$

where $y \in R^{4}$ is the state and $u_{1}, u_{2}, u_{3}, u_{4}$ are the adaptive controllers to be designed.

For the anti-synchronization, the error $e$ is defined as

$$
e_{1}=y_{1}+x_{1}, \quad e_{2}=y_{2}+x_{2}, \quad e_{3}=y_{3}+x_{3}, \quad e_{4}=y_{4}+x_{4}
$$


International Journal of Computer Science, Engineering and Information Technology (IJCSEIT), Vol.3, No.2,April2013

Then we derive the error dynamics as

$$
\begin{aligned}
& \dot{e}_{1}=\alpha\left(e_{2}-e_{1}\right)+e_{4}+u_{1} \\
& \dot{e}_{2}=\delta e_{1}+\gamma e_{2}-y_{1} y_{3}-x_{1} x_{3}+u_{2} \\
& \dot{e}_{3}=-\beta e_{3}+y_{1} y_{2}+x_{1} x_{2}+u_{3} \\
& \dot{e}_{4}=r e_{4}+y_{2} y_{3}+x_{2} x_{3}+u_{4}
\end{aligned}
$$

The adaptive controller to solve the anti-synchronization problem is taken as

$$
\begin{aligned}
& u_{1}=-\hat{\alpha}(t)\left(e_{2}-e_{1}\right)-e_{4}-k_{1} e_{1} \\
& u_{2}=-\hat{\delta}(t) e_{1}-\hat{\gamma}(t) e_{2}+y_{1} y_{3}+x_{1} x_{3}-k_{2} e_{2} \\
& u_{3}=\hat{\beta}(t) e_{3}-y_{1} y_{2}-x_{1} x_{2}-k_{3} e_{3} \\
& u_{4}=-\hat{r}(t) e_{4}-y_{2} y_{3}-x_{2} x_{3}-k_{4} e_{4}
\end{aligned}
$$

In Eq. (31), $k_{i},(i=1,2,3,4)$ are positive gains and $\hat{\alpha}(t), \hat{\beta}(t), \hat{\gamma}(t), \hat{\delta}(t), \hat{r}(t)$ are estimates for the unknown parameters $\alpha, \beta, \gamma, \delta, r$ respectively.

By the substitution of (31) into (30), the error dynamics is obtained as

$$
\begin{aligned}
& \dot{e}_{1}=(\alpha-\hat{\alpha}(t))\left(e_{2}-e_{1}\right)-k_{1} e_{1} \\
& \dot{e}_{2}=(\delta-\hat{\delta}(t)) e_{1}+(\gamma-\hat{\gamma}(t)) e_{2}-k_{2} e_{2} \\
& \dot{e}_{3}=-(\beta-\hat{\beta}(t)) e_{3}-k_{3} e_{3} \\
& \dot{e}_{4}=(r-\hat{r}(t)) e_{4}-k_{4} e_{4}
\end{aligned}
$$

Next, we define the parameter estimation errors as

$$
e_{\alpha}(t)=\alpha-\hat{\alpha}(t), e_{\beta}(t)=\beta-\hat{\beta}(t), e_{\gamma}(t)=\gamma-\hat{\gamma}(t), e_{\delta}(t)=\delta-\hat{\delta}(t), e_{r}(t)=r-\hat{r}(t)
$$

Upon differentiation, we get

$$
\dot{e}_{\alpha}(t)=-\dot{\hat{\alpha}}(t), \dot{e}_{\beta}(t)=-\dot{\hat{\beta}}(t), \dot{e}_{\gamma}(t)=-\dot{\hat{\gamma}}(t), \dot{e}_{\delta}(t)=-\dot{\hat{\delta}}(t), \dot{e}_{r}(t)=-\dot{\hat{r}}(t)
$$

Substituting (33) into the error dynamics (32), we obtain

$$
\begin{aligned}
& \dot{e}_{1}=e_{\alpha}\left(e_{2}-e_{1}\right)-k_{1} e_{1} \\
& \dot{e}_{2}=e_{\delta} e_{1}+e_{\gamma} e_{2}-k_{2} e_{2} \\
& \dot{e}_{3}=-e_{\beta} e_{3}-k_{3} e_{3} \\
& \dot{e}_{4}=e_{r} e_{4}-k_{4} e_{4}
\end{aligned}
$$

We consider the candidate Lyapunov function 
International Journal of Computer Science, Engineering and Information Technology (IJCSEIT), Vol.3, No.2,April2013

$$
V=\frac{1}{2}\left(e_{1}^{2}+e_{2}^{2}+e_{3}^{2}+e_{4}^{2}+e_{\alpha}^{2}+e_{\beta}^{2}+e_{\gamma}^{2}+e_{\delta}^{2}+e_{r}^{2}\right)
$$

Differentiating (36) along the dynamics (34) and (35), we obtain

$$
\begin{aligned}
\dot{V}= & -k_{1} e_{1}^{2}-k_{2} e_{2}^{2}-k_{3} e_{3}^{2}-k_{4} e_{4}^{2}+e_{\alpha}\left[e_{1}\left(e_{2}-e_{1}\right)-\dot{\hat{\alpha}}\right]+e_{\beta}\left(-e_{3}^{2}-\dot{\hat{\beta}}\right) \\
& +e_{\gamma}\left(e_{2}^{2}-\dot{\hat{\gamma}}\right)+e_{\delta}\left(e_{1} e_{2}-\dot{\hat{\delta}}\right)+e_{r}\left(e_{4}^{2}-\dot{\hat{r}}\right)
\end{aligned}
$$

In view of (37), we choose the following parameter update law:

$$
\begin{aligned}
& \dot{\hat{\alpha}}=e_{1}\left(e_{2}-e_{1}\right)+k_{5} e_{a} \\
& \dot{\hat{\beta}}=-e_{3}^{2}+k_{6} e_{\beta} \\
& \dot{\hat{\gamma}}=e_{2}^{2}+k_{7} e_{\gamma} \\
& \dot{\hat{\delta}}=e_{1} e_{2}+k_{8} e_{\delta} \\
& \dot{\hat{r}}=e_{4}^{2}+k_{9} e_{r}
\end{aligned}
$$

Next, we prove the following main result of this section.

Theorem 5.1 The adaptive control law defined by Eq. (31) along with the parameter update law defined by Eq. (38), where $k_{i},(i=1,2, \ldots, 9)$ are positive constants, render global and exponential anti-synchronization of the identical hyperchaotic Li systems (27) and (28) with unknown parameters for all initial conditions $x(0), y(0) \in R^{4}$. In addition, the parameter estimation errors $e_{\alpha}(t), e_{\beta}(t), e_{\gamma}(t), e_{\delta}(t), e_{r}(t)$ globally and exponentially converge to zero for all initial conditions.

Proof. The proof is via Lyapunov stability theory [25] by taking $V$ defined by Eq. (36) as the candidate Lyapunov function. Substituting the parameter update law (38) into (37), we get

$$
\dot{V}(e)=-k_{1} e_{1}^{2}-k_{2} e_{2}^{2}-k_{3} e_{3}^{2}-k_{4} e_{4}^{2}-k_{5} e_{\alpha}^{2}-k_{6} e_{\beta}^{2}-k_{7} e_{\gamma}^{2}-k_{8} e_{\delta}^{2}-k_{9} e_{r}^{2}
$$

which is a negative definite function on $R^{9}$. This completes the proof.

Next, we demonstrate our adaptive anti-synchronization results with MATLAB simulations. The classical fourth order R-K method with time-step $h=10^{-8}$ has been used to solve the hyperchaotic Li systems (27) and (28) with the adaptive controller defined by (31) and parameter update law defined by (38). The feedback gains in the adaptive controller (31) are taken as $k_{i}=5,(i=1, \ldots, 9)$.

The parameters of the hyperchaotic Li systems are taken as in the hyperchaotic case, i.e.

$$
\alpha=35, \quad \beta=3, \quad \gamma=12, \quad \delta=7, \quad r=0.58
$$

For simulations, the initial conditions of the drive system (27) are taken as 
International Journal of Computer Science, Engineering and Information Technology (IJCSEIT), Vol.3, No.2,April2013

$$
x_{1}(0)=7, \quad x_{2}(0)=-26, \quad x_{3}(0)=12, \quad x_{4}(0)=14
$$

Also, the initial conditions of the response system (28) are taken as

$$
y_{1}(0)=-21, \quad y_{2}(0)=28, \quad y_{3}(0)=-18, \quad y_{4}(0)=29
$$

Also, the initial conditions of the parameter estimates are taken as

$$
\hat{\alpha}(0)=7, \quad \hat{\beta}(0)=15, \quad \hat{\gamma}(0)=5, \quad \hat{\delta}(0)=4, \quad \hat{r}(0)=-3
$$

Figure 6 depicts the anti-synchronization of the identical hyperchaotic Li systems.

Figure 7 depicts the time-history of the anti-synchronization errors $e_{1}, e_{2}, e_{3}, e_{4}$.

Figure 8 depicts the time-history of the parameter estimation errors $e_{\alpha}, e_{\beta}, e_{\gamma}, e_{\delta}, e_{r}$.

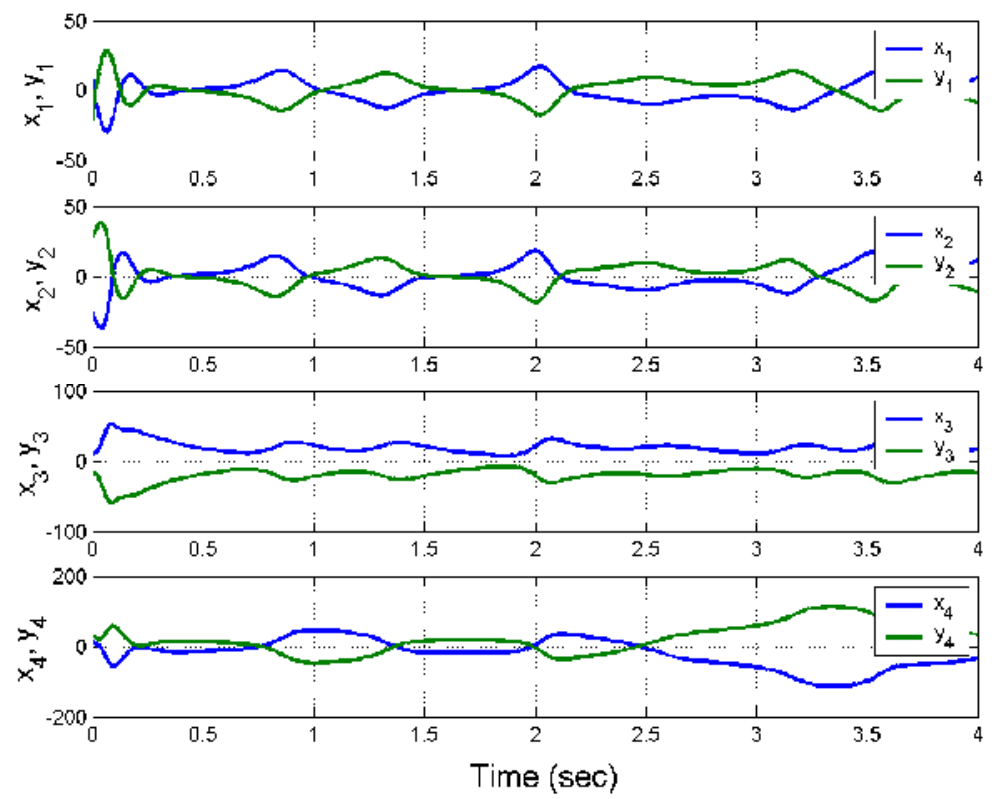

Figure 6. Anti-Synchronization of Identical Hyperchaotic Li Systems 


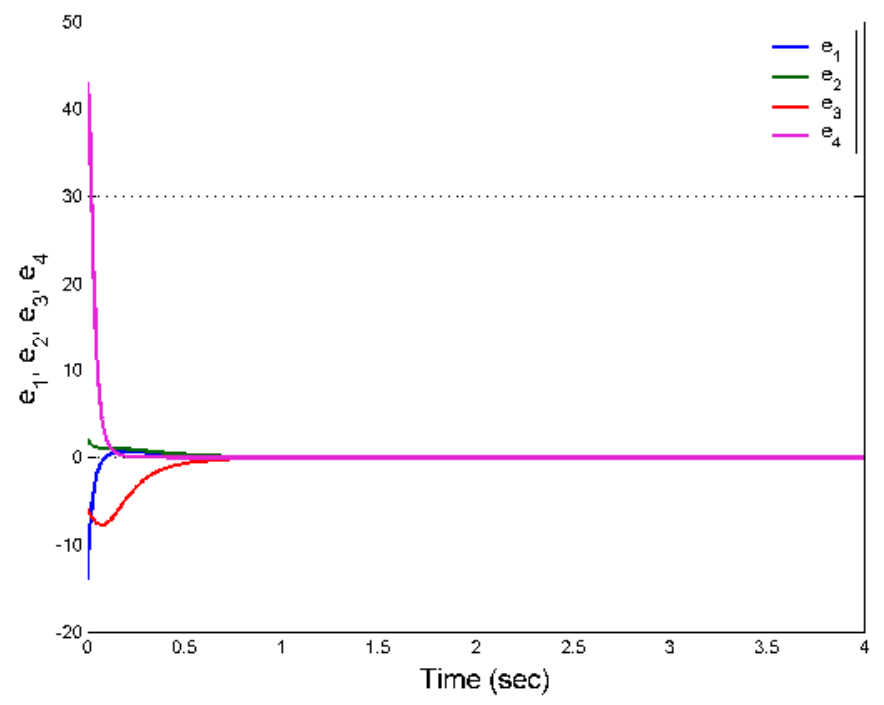

Figure 7. Time-History of the Anti-Synchronization Errors $e_{1}, e_{2}, e_{3}, e_{4}$

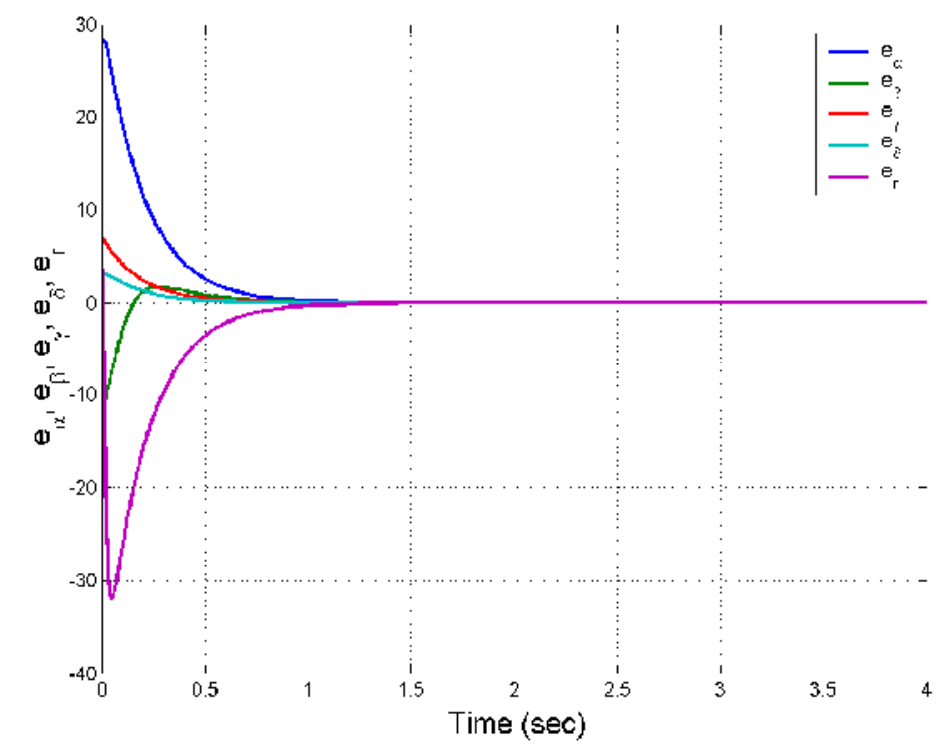

Figure 8. Time-History of the Parameter Estimation Errors $e_{\alpha}, e_{\beta}, e_{\gamma}, e_{\delta}, e_{r}$

\section{ANTI-SYNCHRONIZATION OF HYPERCHAOTIC WANG AND HYPERCHAOTIC Li SYSTEMS VIA ADAPTIVE CONTROL}

In this section, we derive new results for designing a controller for the anti-synchronization of non-identical hyperchaotic Wang system (2009) and hyperchaotic Li system (2005) with unknown parameters via adaptive control.

The drive system is the hyperchaotic Wang dynamics given by 
International Journal of Computer Science, Engineering and Information Technology (IJCSEIT), Vol.3, No.2,April2013

$$
\begin{aligned}
& \dot{x}_{1}=a\left(x_{2}-x_{1}\right)+x_{2} x_{3} \\
& \dot{x}_{2}=c x_{1}-x_{1} x_{3}-x_{2}-0.5 x_{4} \\
& \dot{x}_{3}=-d x_{3}+x_{1} x_{2} \\
& \dot{x}_{4}=b x_{4}+0.5 x_{1} x_{3}
\end{aligned}
$$

where $a, b, c, d$ are unknown parameters of the system and $x \in R^{4}$ is the state. The response system is the controlled hyperchaotic Li dynamics given by

$$
\begin{aligned}
& \dot{y}_{1}=\alpha\left(y_{2}-y_{1}\right)+y_{4}+u_{1} \\
& \dot{y}_{2}=\delta y_{1}-y_{1} y_{3}+\gamma y_{2}+u_{2} \\
& \dot{y}_{3}=-\beta y_{3}+y_{1} y_{2}+u_{3} \\
& \dot{y}_{4}=y_{2} y_{3}+r y_{4}+u_{4}
\end{aligned}
$$

where $\alpha, \beta, \gamma, \delta, r$ are unknown parameters, $y \in R^{4}$ is the state and $u_{1}, u_{2}, u_{3}, u_{4}$ are the adaptive controllers to be designed.

For the anti-synchronization, the error $e$ is defined as

$$
e_{1}=y_{1}+x_{1}, \quad e_{2}=y_{2}+x_{2}, \quad e_{3}=y_{3}+x_{3}, \quad e_{4}=y_{4}+x_{4}
$$

Then we derive the error dynamics as

$$
\begin{aligned}
& \dot{e}_{1}=a\left(x_{2}-x_{1}\right)+\alpha\left(y_{2}-y_{1}\right)+y_{4}+x_{2} x_{3}+u_{1} \\
& \dot{e}_{2}=c x_{1}+\delta y_{1}-x_{2}+\gamma y_{2}-0.5 x_{4}-x_{1} x_{3}-y_{1} y_{3}+u_{2} \\
& \dot{e}_{3}=-d x_{3}-\beta y_{3}+y_{1} y_{2}+x_{1} x_{2}+u_{3} \\
& \dot{e}_{4}=b x_{4}+r y_{4}+0.5 x_{1} x_{3}+y_{2} y_{3}+u_{4}
\end{aligned}
$$

The adaptive controller to solve the anti-synchronization problem is taken as

$$
\begin{aligned}
& u_{1}=-\hat{a}(t)\left(x_{2}-x_{1}\right)-\hat{\alpha}(t)\left(y_{2}-y_{1}\right)-y_{4}-x_{2} x_{3}-k_{1} e_{1} \\
& u_{2}=-\hat{c}(t) x_{1}-\hat{\delta}(t) y_{1}+x_{2}-\hat{\gamma}(t) y_{2}+0.5 x_{4}+x_{1} x_{3}+y_{1} y_{3}-k_{2} e_{2} \\
& u_{3}=\hat{d}(t) x_{3}+\hat{\beta}(t) y_{3}-y_{1} y_{2}-x_{1} x_{2}-k_{3} e_{3} \\
& u_{4}=-\hat{b}(t) x_{4}-\hat{r}(t) y_{4}-0.5 x_{1} x_{3}-y_{2} y_{3}-k_{4} e_{4}
\end{aligned}
$$

In Eq. (44), $k_{i},(i=1,2,3,4)$ are positive gains and $\hat{a}(t), \hat{b}(t), \hat{c}(t), \hat{d}(t), \hat{\alpha}(t), \hat{\beta}(t), \hat{\gamma}(t)$, $\hat{\delta}(t), \hat{r}(t)$ are estimates for the unknown parameters $a, b, c, d, \alpha, \beta, \gamma, \delta, r$ respectively. By the substitution of (44) into (43), the error dynamics is obtained as

$$
\begin{aligned}
& \dot{e}_{1}=(a-\hat{a}(t))\left(x_{2}-x_{1}\right)+(\alpha-\hat{\alpha}(t))\left(y_{2}-y_{1}\right)-k_{1} e_{1} \\
& \dot{e}_{2}=(c-\hat{c}(t)) x_{1}+(\delta-\hat{\delta}(t)) y_{1}+(\gamma-\hat{\gamma}(t)) y_{2}-k_{2} e_{2} \\
& \dot{e}_{3}=-(d-\hat{d}(t)) x_{3}-(\beta-\hat{\beta}(t)) y_{3}-k_{3} e_{3} \\
& \dot{e}_{4}=(b-\hat{b}(t)) x_{4}+(r-\hat{r}(t)) y_{4}-k_{4} e_{4}
\end{aligned}
$$


International Journal of Computer Science, Engineering and Information Technology (IJCSEIT), Vol.3, No.2,April2013

Next, we define the parameter estimation errors as

$$
\begin{aligned}
& e_{a}(t)=a-\hat{a}(t), e_{b}(t)=b-\hat{b}(t), e_{c}(t)=c-\hat{c}(t), e_{d}(t)=d-\hat{d}(t) \\
& e_{\alpha}(t)=\alpha-\hat{\alpha}(t), e_{\beta}(t)=\beta-\hat{\beta}(t), e_{\gamma}(t)=\gamma-\hat{\gamma}(t), e_{\delta}(t)=\delta-\hat{\delta}(t), e_{r}(t)=r-\hat{r}(t)
\end{aligned}
$$

Upon differentiation, we get

$$
\begin{aligned}
& \dot{e}_{a}(t)=-\dot{\hat{a}}(t), \dot{e}_{b}(t)=-\dot{\hat{b}}(t), \dot{e}_{c}(t)=-\dot{\hat{c}}(t), \dot{e}_{d}(t)=-\dot{\hat{d}}(t) \\
& \dot{e}_{\alpha}(t)=-\dot{\hat{\alpha}}(t), \dot{e}_{\beta}(t)=-\dot{\hat{\beta}}(t), \dot{e}_{\gamma}(t)=-\dot{\hat{\gamma}}(t), \dot{e}_{\delta}(t)=-\dot{\hat{\delta}}(t), \dot{e}_{r}(t)=-\dot{\hat{r}}(t)
\end{aligned}
$$

Substituting (46) into the error dynamics (45), we obtain

$$
\begin{aligned}
& \dot{e}_{1}=e_{a}\left(x_{2}-x_{1}\right)+e_{\alpha}\left(y_{2}-y_{1}\right)-k_{1} e_{1} \\
& \dot{e}_{2}=e_{c} x_{1}+e_{\delta} y_{1}+e_{\gamma} y_{2}-k_{2} e_{2} \\
& \dot{e}_{3}=-e_{d} x_{3}-e_{\beta} y_{3}-k_{3} e_{3} \\
& \dot{e}_{4}=e_{b} x_{4}+e_{r} y_{4}-k_{4} e_{4}
\end{aligned}
$$

We consider the candidate Lyapunov function

$$
V=\frac{1}{2}\left(e_{1}^{2}+e_{2}^{2}+e_{3}^{2}+e_{4}^{2}+e_{a}^{2}+e_{b}^{2}+e_{c}^{2}+e_{d}^{2}+e_{\alpha}^{2}+e_{\beta}^{2}+e_{\gamma}^{2}+e_{\delta}^{2}+e_{r}^{2}\right)
$$

Differentiating (49) along the dynamics (47) and (48), we obtain

$$
\begin{aligned}
\dot{V}= & -k_{1} e_{1}^{2}-k_{2} e_{2}^{2}-k_{3} e_{3}^{2}-k_{4} e_{4}^{2}+e_{a}\left[e_{1}\left(x_{2}-x_{1}\right)-\dot{\hat{a}}\right]+e_{b}\left(e_{4} x_{4}-\dot{\hat{b}}\right)+e_{c}\left(e_{2} x_{1}-\dot{\hat{c}}\right) \\
& +e_{d}\left(-e_{3} x_{3}-\dot{\hat{d}}\right)+e_{\alpha}\left[e_{1}\left(y_{2}-y_{1}\right)-\dot{\hat{\alpha}}\right]+e_{\beta}\left(-e_{3} y_{3}-\dot{\hat{\beta}}\right) \\
& +e_{\gamma}\left(e_{2} y_{2}-\dot{\hat{\gamma}}\right)+e_{\delta}\left(e_{2} y_{1}-\dot{\hat{\delta}}\right)+e_{r}\left(e_{4} y_{4}-\dot{\hat{r}}\right)
\end{aligned}
$$

In view of (50), we choose the following parameter update law:

$$
\begin{array}{ll}
\dot{\hat{a}}=e_{1}\left(x_{2}-x_{1}\right)+k_{5} e_{a}, & \dot{\hat{\alpha}}=e_{1}\left(y_{2}-y_{1}\right)+k_{9} e_{a} \\
\dot{\hat{b}}=e_{4} x_{4}+k_{6} e_{b}, & \dot{\hat{\beta}}=-e_{3} y_{3}+k_{10} e_{\beta} \\
\dot{\hat{c}}=e_{2} x_{1}+k_{7} e_{c}, & \dot{\hat{\gamma}}=e_{2} y_{2}+k_{11} e_{\gamma} \\
\dot{\hat{d}}=-e_{3} x_{3}+k_{8} e_{d}, & \dot{\hat{\delta}}=e_{2} y_{1}+k_{12} e_{\delta} \\
\dot{\hat{r}}=e_{4} y_{4}+k_{13} e_{r} &
\end{array}
$$

Next, we prove the following main result of this section. 
International Journal of Computer Science, Engineering and Information Technology (IJCSEIT), Vol.3, No.2,April2013

Theorem 6.1 The adaptive control law defined by Eq. (44) along with the parameter update law defined by Eq. (51), where $k_{i},(i=1,2, \ldots, 13)$ are positive constants, render global and exponential anti-synchronization of the non-identical hyperchaotic Wang system (40) and hyperchaotic Li system (41) with unknown parameters for all initial conditions $x(0), y(0) \in R^{4}$. In addition, all the parameter estimation errors globally and exponentially converge to zero for all initial conditions.

Proof. The proof is via Lyapunov stability theory [25] by taking $V$ defined by Eq. (49) as the candidate Lyapunov function. Substituting the parameter update law (51) into (50), we get

$$
\begin{aligned}
\dot{V}(e)= & -k_{1} e_{1}^{2}-k_{2} e_{2}^{2}-k_{3} e_{3}^{2}-k_{4} e_{4}^{2}-k_{5} e_{a}^{2}-k_{6} e_{b}^{2}-k_{7} e_{c}^{2}-k_{8} e_{d}^{2} \\
& -k_{9} e_{\alpha}^{2}-k_{10} e_{\beta}^{2}-k_{11} e_{\gamma}^{2}-k_{12} e_{\delta}^{2}-k_{13} e_{r}^{2}
\end{aligned}
$$

which is a negative definite function on $R^{13}$. This completes the proof.

Next, we demonstrate our adaptive anti-synchronization results with MATLAB simulations. The classical fourth order R-K method with time-step $h=10^{-8}$ has been used to solve the hyperchaotic systems (40) and (41) with the adaptive controller defined by (44) and parameter update law defined by (51).

The feedback gains in the adaptive controller (44) are taken as $k_{i}=5,(i=1, \ldots, 13)$.

The parameters of the hyperchaotic Wang and Li systems are taken as in the hyperchaotic case, i.e.

$$
a=40, \quad b=1.7, \quad c=88, d=3, \alpha=35, \quad \beta=3, \quad \gamma=12, \delta=7, \quad r=0.58
$$

For simulations, the initial conditions of the drive system (40) are taken as

$$
x_{1}(0)=12, \quad x_{2}(0)=-34, \quad x_{3}(0)=31, \quad x_{4}(0)=14
$$

Also, the initial conditions of the response system (41) are taken as

$$
y_{1}(0)=-25, \quad y_{2}(0)=18, \quad y_{3}(0)=-12, \quad y_{4}(0)=29
$$

Also, the initial conditions of the parameter estimates are taken as

$$
\begin{array}{ll}
\hat{a}(0)=21, & \hat{b}(0)=14, \quad \hat{c}(0)=-26, \quad \hat{d}(0)=16 \\
\hat{\alpha}(0)=17, \quad \hat{\beta}(0)=-22 \quad \hat{\gamma}(0)=15, \quad \hat{\delta}(0)=11, \quad \hat{r}(0)=-7
\end{array}
$$

Figure 9 depicts the anti-synchronization of the hyperchaotic Wang and hyperchaotic Li systems. Figure 10 depicts the time-history of the anti-synchronization errors $e_{1}, e_{2}, e_{3}, e_{4}$.

Figure 11 depicts the time-history of the parameter estimation errors $e_{a}, e_{b}, e_{c}, e_{d}$.

Figure 12 depicts the time-history of the parameter estimation errors $e_{\alpha}, e_{\beta}, e_{\gamma}, e_{\delta}, e_{r}$. 

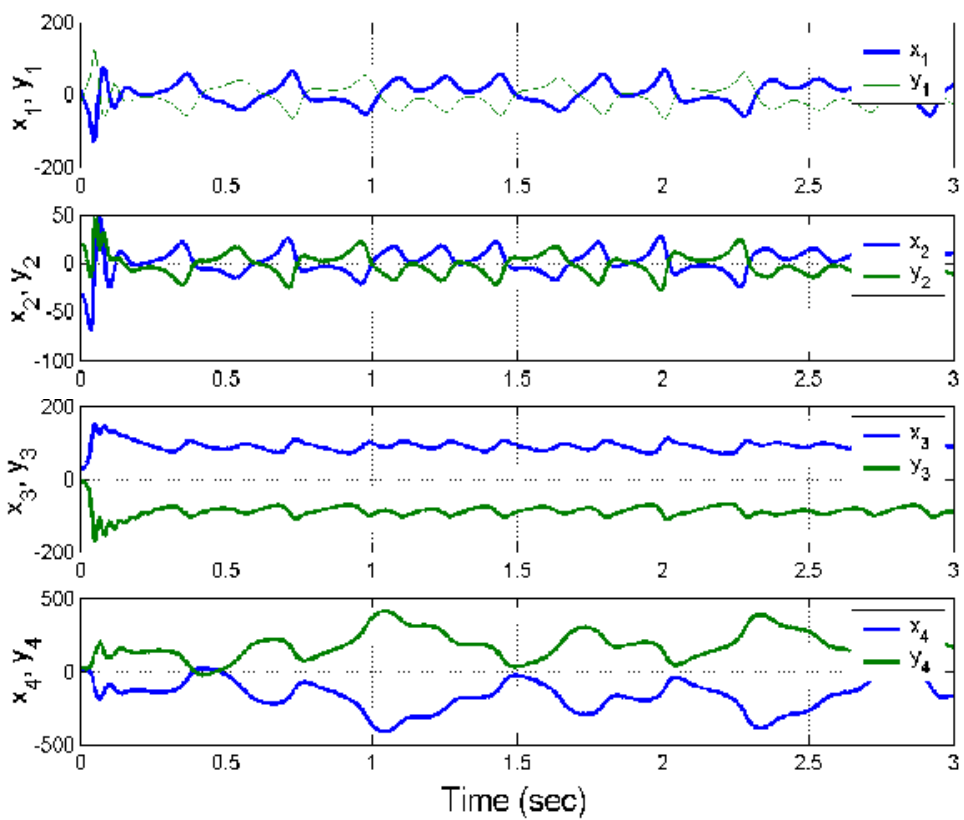

Figure 9. Anti-Synchronization of Hyperchaotic Wang and Hyperchaotic Li Systems

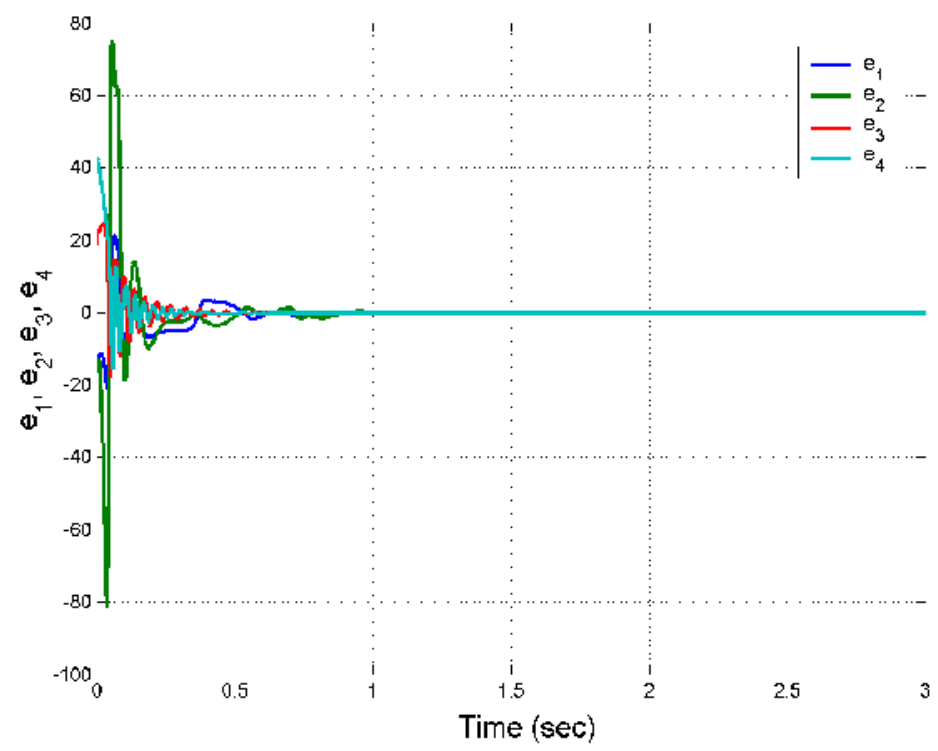

Figure 10. Time-History of the Anti-Synchronization Errors $e_{1}, e_{2}, e_{3}, e_{4}$ 


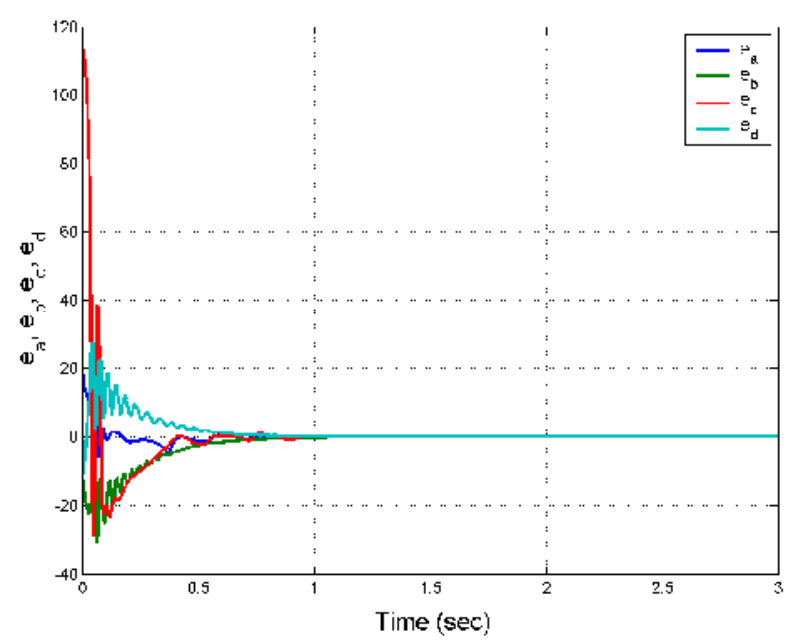

Figure 11. Time-History of the Parameter Estimation Errors $e_{a}, e_{b}, e_{c}, e_{d}$

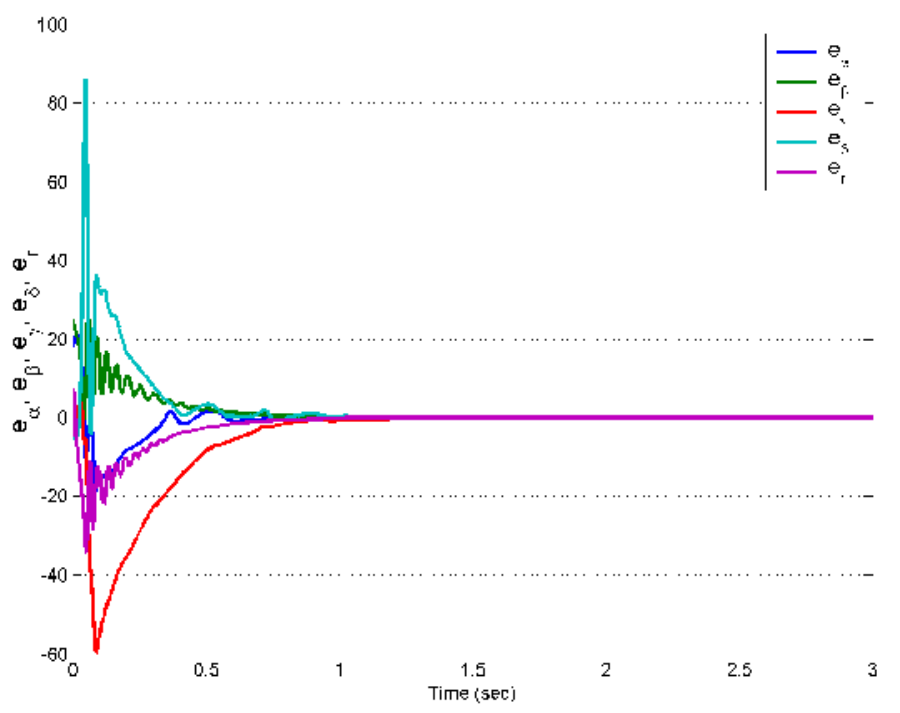

Figure 12. Time-History of the Parameter Estimation Errors $e_{\alpha}, e_{\beta}, e_{\gamma}, e_{\delta}, e_{r}$

\section{Conclusions}

This paper has used adaptive control theory and Lyapunov stability theory so as to solve the antisynchronization problem for the anti-synchronization of hyperchaotic Wang system (2008) and hyperchaotic Li system (2005) with unknown parameters. Hyperchaotic systems are chaotic systems with two or more positive Lyapunov exponents and they have viable applications like chemical reactions, neural networks, secure communication, data encryption, neural networks, etc. MATLAB simulations were depicted to illustrate the various adaptive anti-synchronization results derived in this paper for the hyperchaotic Wang and Li systems. 


\section{REFERENCES}

[1] Misra, A.P., Ghosh, D. \& Chowdhury, A.R. (2008) "A novel hyperchaos in the quantum Zakharov system for plasmas,” Physics Letters A, Vol. 372, No. 9, pp 1469-1476.

[2] Eiswirth, M., Kruel, T.M., Ertl, G. \& Schneider, F.W. (1992) "Hyperchaos in a chemical reaction," Chemical Physics Letters, Vol. 193, No. 4, pp 305-310.

[3] Huang, Y. \& Yang, X.S. (2006) "Hyperchaos and bifurcation in a new class of four-dimensional Hopfield neural networks," Neurocomputing, Vol. 69, pp 13-15.

[4] Machado, L.G., Savi, M.A. \& Pacheco, P.M.C.L. (2003) "Nonlinear dynamics and chaos in coupled shape memory oscillators," International Journal of Solids and Structures, Vol. 40, No. 19, pp. 51395156.

[5] Prokhorov, M.D. \& Ponomarenko, V.I. (2008) "Encryption and decryption of information in chaotic communication systems governed by delay-differential equations," Chaos, Solitons \& Fractals, Vol. 35, No. 5, pp 871-877.

[6] Tao, Y. (1999) "Chaotic secure communication systems - history and new results", Telecommun. Review, Vol. 9, pp 597-634.

[7] Li, C., Liao, X. \& Wong, K.W. (2005) "Lag synchronization of hyperchaos with applications to secure communications," Chaos, Solitons \& Fractals, Vol. 23, No. 1, pp 183-193.

[8] Nana, B., Woafo, P. \& Domngang, S. (2009) "Chaotic synchronization with experimental applications to secure communications”, Comm. Nonlinear Sci. Numerical Simulation, Vol. 14, No. 5, pp 2266-2276.

[9] Sundarapandian, V. \& Karthikeyan, R. (2011) “Anti-synchronization of Pan and Liu chaotic systems by active nonlinear control," International Journal of Engineering Science and Technology, Vol. 3, No. 5, pp 3596-3604.

[10] Huang, L. Feng, R. \& Wang, M. (2004) "Synchronization of chaotic systems via nonlinear control," Physics Letters A, Vol. 320, No. 4, pp 271-275.

[11] Lei, Y., Xu, W. \& Zheng, H. (2005) "Synchronization of two chaotic nonlinear gyros using active control,” Physics Letters A, Vol. 343, pp 153-158.

[12] Sarasu, P. \& Sundarapandian, V. (2011) “Active controller design for generalized projective synchronization of four-scroll chaotic systems", International Journal of System Signal Control and Engineering Application, Vol. 4, No. 2, pp 26-33.

[13] Sarasu, P. \& Sundarapandian, V. (2012) "Generalized projective synchronization of two-scroll systems via adaptive control," International Journal of Soft Computing, Vol. 7, No. 4, pp 146-156.

[14] Sundarapandian, V. (2012) "Adaptive control and synchronization of a generalized Lotka-Volterra system," Vol. 1, No. 1, pp 1-12.

[15] Sundarapandian, V. (2013) "Adaptive controller and synchronizer design for hyperchaotic Zhou system with unknown parameters," Vol. 1, No. 1, pp 18-32.

[16] Bowong, S. \& Kakmeni, F.M.M. (2004) "Synchronization of uncertain chaotic systems via backstepping approach," Chaos, Solitons \& Fractals, Vol. 21, No. 4, pp 999-1011.

[17] Suresh, R. \& Sundarapandian, V. (2012) "Hybrid synchronization of n-scroll Chua and Lur'e chaotic systems via backstepping control with novel feedback", Arch. Control Sciences, Vol. 22, No. 3, pp 255-278.

[18] Suresh, R, \& Sundarapandian, V. (2012) "Global chaos synchronization of WINDMI and Coullet chaotic systems by backstepping control”, Far East J. Math. Sciences, Vol. 67, No. 2, pp 265-287.

[19] Sundarapandian, V. (2013) "Anti-synchronizing backstepping design for Arneodo chaotic system", International Journal on Bioinformatics and Biosciences, Vol. 3, No. 1, pp 21-33.

[20] Senejohnny, D.M. \& Delavari, H. (2012) "Active sliding observer scheme based fractional chaos synchronization," Comm. Nonlinear Sci. Numerical Simulation, Vol. 17, No. 11, pp 4373-4383.

[21] Sundarapandian, V. (2012) "Anti-synchronization of hyperchaotic Xu systems via sliding mode control”, International Journal of Embedded Systems, Vol. 2, No. 2, pp 51-61.

[22] Sundarapandian, V. (2013) "Anti-synchronizing sliding controller design for identical Pan systems," International Journal of Computational Science and Information Technology, Vol. 1, No. 1, pp 1-9.

[23] Wang, J. \& Chen, Z. (2008) “A novel hyperchaotic system and its complex dynamics," International Journal of Bifurcation and Chaos, Vol. 18, No. 11, pp 3309-3324.

[24] Li, Y.,Tang, W.K.S. \& Chen, G. (2005) "Generating hyperchaos via state feedback control," International Journal of Bifurcation and Chaos, Vol. 15, No. 10, pp 3367-3375.

[25] Hahn, W. (1967) The Stability of Motion, Springer, Berlin. 


\section{Author}

Dr. V. Sundarapandian earned his D.Sc. in Electrical and Systems Engineering from Washington University, St. Louis, USA in May 1996. He is Professor and Dean of the R \& D Centre at Vel Tech Dr. RR \& Dr. SR Technical University, Chennai, Tamil Nadu, India. So far, he has published over 300 research works in refereed international journals. He has also published over 200 research papers in National and International Conferences. He has delivered Key Note Addresses at many International Conferences with IEEE and Springer Proceedings. He is an India Chair of AIRCC. He is the Editor-in-Chief of the AIRCC Control Journals - International Journal of Instrumentation and Control Systems, International Journal of Control

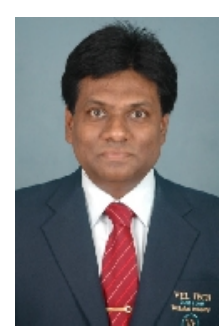
Theory and Computer Modeling, International Journal of Information Technology, Control and Automation, International Journal of Chaos, Control, Modelling and Simulation, and International Journal of Information Technology, Modeling and Computing. His research interests are Control Systems, Chaos Theory, Soft Computing, Operations Research, Mathematical Modelling and Scientific Computing. He has published four text-books and conducted many workshops on Scientific Computing, MATLAB and SCILAB. 